\title{
Minimax design of recursive digital filters with a lattice denominator
}

J.-H.Lee

S.-Y.Ku

\begin{abstract}
The authors deal with the problem of minimax recursive digital filter design with a lattice structure for the denominator. The design problem is formulated so that the coefficients for the numerator and denominator of a recursive filter can be found by solving the best linear complex Chebyshev approximation (LCCA). A design technique based on the weighted leastsquares algorithm previously proposed by one of the authors is then developed for solving the resulting LCCA problem. During the design process, this technique finds the tap coefficients for the numerator and the reflection coefficients for the denominator simultaneously. The stability of the designed recursive filter is ensured by incorporating an efficient stabilisation procedure to make all of the reflection coefficient values fall between -1 and +1 . Computer simulations show that the proposed technique provides better design results than existing techniques.
\end{abstract}

\section{Introduction}

In many applications, such as in the design of digital phase networks and of minimum phase systems, digital filters with constant group delay phase other than exactly linear phase are usually required. Therefore, a complex approximation problem arises when we design a filter to approximate arbitrary magnitude, phase or group delay.

Several algorithms have been proposed to solve the problem of designing recursive filters with arbitrary magnitude and phase responses optimal in the minimax sense. Because the design problem is basically a rational Chebyshev approximation problem in the complex domain, it is not easy to solve this approximation problem and hence there are no systematic approaches for finding the best approximation solution in the literature. In general, researchers resort to a local best approximation (LBA) solution instead of the global best approximation (GBA) solution for this considered problem. Linear programning (LP) based algorithms have been used in [1] for dealing with this

\section{(C) IEE, 1996}

IEE Proceedings online no. 19960905

Paper received 19th March 1996

The authors are with the Department of Electrical Engineering, National Taiwan University, Taipei 106, Taiwan design problem. The objective function resulted from the formulation employs the frequency response of the denominator of the filter as the weighting function. Moreover, a very strict stability constraint is also utilised to ensure the stability of the designed recursive filter. This technique cannot provide satisfactory design results. A multiple criterion optimisation technique has been presented in [2] for this design. It utilised the sequential quadratic programming algorithm of [3] to solve the resulting optimisation problem. In general, the capabilities of this design technique are limited when recursive filters with higher order are required. A recent technique is proposed by [4]. However, it employs a linear complex Chebyshev approximation (LCCA) tool presented in [5] to modify the original Ellacott-Williams (EW) algorithm of [6]. An LP based method is used to solve the resulting LCCA problem. This technique does not guarantee that the best approximation solution can be obtained even in the local optimal sense. Moreover, it is not an efficient way to use any LP based algorithms to solve the resulting LCCA problem because huge computational load is inevitably required.

In this paper, we present a technique for designing a recursive filter with a lattice structure for the denominator in the minimax sense. The related design problem is first formulated so that the coefficients for the numerator and the reflection coefficients for the denominator of a recursive filter can be found by solving a problem of the best linear complex Chebȳshev approximation (LCCA). To efficiently solve the resulting LCCA problem induced by the minimax design of recursive digital filters with arbitrary magnitude and phase responses, a design technique based on the weighted least squares (WLS) algorithm of [7] is then developed. During the design process, this technique finds the tap coefficients for the numerator and the reflection coefficients for the denominator simultaneously without resorting to any LP based algorithm. It ensures the stability of the designed recursive filter by incorporating an efficient stabilisation procedure to make the magnitude of each reflection coefficient within -1 and +1 . Computer simulations show that the proposed technique can provide a more satisfactory design than the existing techniques for each design example.

\section{Formulation of the design problem}

Let the transfer function of the recursive digital filter with order $M / N$ (i.e. $M$ zeros and $N$ poles) be given by $H(z)=A(z) / B_{N}(z)$, where $A(z)$ is an $M$ th-order polyno- 
mial with tap coefficient vector $\mathbf{A}=\left[a_{0}, a_{1}, \ldots, a_{M}\right]^{T}$, $B_{N}(z)$ is an $N$ th-order FIR lattice filter with reflection coefficient vector $\mathbf{K}=\left[k_{1}, k_{2}, \ldots, k_{N}\right]^{T}$. The superscript $T$ denotes the transpose operation. Fig. 1 shows the system structure for $B_{N}(z)$ which can be obtained from the following recursive formula [8]:

$$
\begin{aligned}
& B_{0}(z)=Q_{0}(z)=1 \\
& B_{n}(z)=B_{n-1}(z)+k_{n} z^{-1} Q_{n-1}(z) \\
& Q_{n}(z)=k_{n} B_{n-1}(z)+z^{-1} Q_{n-1}(z)
\end{aligned}
$$

The design problem is to find the tap and reflection coefficients $\left\{a_{m}, k_{n}\right\}$ such that the stable filter $H(z)$ has a frequency response $H\left(e^{j \omega}\right)$ optimally approximating a desired complex frequency response $D\left(e^{j \omega}\right)$ in the mini$\max$ sense over a set $\mathrm{S}$ which consists of the considered frequency regions. That is, we want to find a polynomial $A(z)$ and a lattice system $B_{N}(z)$ such that the following weighted Chebyshev error,

$$
\left\|W_{r}\left(e^{j \omega}\right)\left(D\left(e^{j \omega}\right)-\frac{A\left(e^{j \omega}\right)}{B_{N}\left(e^{j \omega}\right)}\right)\right\|=\max \left|W_{r}\left(e^{j \omega}\right)\left(D\left(e^{j \omega}\right)-\frac{A\left(e^{j \omega}\right)}{B_{N}\left(e^{j \omega}\right)}\right)\right| \forall \omega \in S
$$

is minimised, where $W_{r}\left(e^{j \omega}\right)$ is a non-negative weighting function defined on $S$ for putting relative weights between different frequency bands. eqn. 2 reveals that the design problem is a rational Chebyshev approximation problem in the complex domain like that considered in [1]. To find the solution numerically requires evaluation of the error of eqn. 2 on a set of discrete frequency points. Let $S_{d}=\left\{\omega_{1}, \omega_{2}, \ldots, \omega_{L}\right\}$ be a dense grid of frequency points linearly distributed over $\mathrm{S}$ with $\omega_{i+1}>\omega_{i}$ for $0<i<L-1$. Then, we consider the optimisation problem of eqn. 2 on $S_{d}$ and rewrite eqn. 2 as follows:

minimise $\max \left|W_{r}\left(e^{j \omega_{i}}\right)\left(D\left(e^{j \omega_{i}}\right)-\frac{A\left(e^{j \omega_{i}}\right)}{B_{N}\left(e^{j \omega_{i}}\right)}\right)\right| \forall \omega_{i} \in S_{d}$

Eqn. 3 represents a rational complex Chebysher approximation (RCCA) problem. In the literature, there are no systematic approaches developed for finding the GBA solution for eqn. 3. Usually, design techniques are developed based on finding an LBA solution instead of the GBA solution. To achieve this design task, we first present an efficient method for solving an LCCA problem in the following Section.

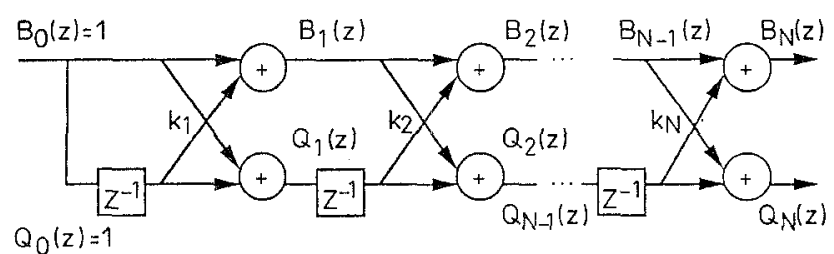

Fig. 1 Lattice structure for an Nth-order FIR lattice filter

\section{An efficient method for solving LCCA problems}

Let $\mathbf{X}$ be an $L \times K$ complex-valued matrix with $L>K$ and $\mathbf{d}$ be an $L \times 1$ complex-valued vector. An LCCA problem can be formulated as follows:

$$
\text { minimise }\|\mathbf{d}-\mathbf{X a}\|
$$

where $\mathbf{a}$ is a real-valued parameter vector of size $K \times 1$. Typically, finding the optimal solution for eqn. 4 requires solving the Chebyshev optimisation of an overdetermined system of linear complex equations. Based on the WLS algorithm of [7], the solution for the optimisation problem of eqn. 4 can be found by solving the following minimisation problem:

$$
\text { minimise } J=(\mathrm{X} \mathbf{a}-\mathrm{d})^{H} \mathbf{W}(\mathrm{Xa}-\mathrm{d})
$$

where the diagonal matrix $\mathbf{W}$ denotes the least-squares error weighting matrix. The superscript $H$ denotes the complex conjugate transpose. In [7], an efficient systematic approach was presented for adjusting $\mathbf{W}$ iteratively during the minimisation process to obtain the Chebyshev solution. It is easy to show that the optimal parameter a which minimises eqn. 5 is given by

$$
\mathbf{a}=\left(\mathbf{X}^{H} \mathbf{W X}\right)^{-1} \mathbf{X}^{H} \mathbf{W d}
$$

However, eqn. 6 cannot guarantee that the resulting a is a real-valued vector. To obtain a real-valued parameter vector a which is still a WLS solution, we consider the following minimisation problem:

minimise $J_{1}=\left(\mathbf{X}_{1} \mathbf{a}-\mathbf{d}_{r}\right)^{T} \mathbf{W}\left(\mathbf{X}_{1} \mathbf{a}-\mathbf{d}_{r}\right)+\left(\mathbf{X}_{i} \mathbf{a}-\mathbf{d}_{i}\right)^{T} \mathbf{W}\left(\mathbf{X}_{i} \mathbf{a}-\mathbf{d}_{i}\right)$

where $\mathbf{X}_{r}$ and $\mathbf{X}_{i}$ are the real and imaginary parts of $\mathbf{X}$, respectively. $\mathbf{d}_{r}$ and $\mathbf{d}_{i}$ are the real and imaginary parts of $\mathbf{d}$, respectively. Again, the optimal solution for eqn. 7 can be found easily as follows:

$$
\mathbf{a}=\left(\mathbf{X}_{r}^{T} \mathbf{W} \mathbf{X}_{r}+\mathbf{X}_{i}^{T} \mathbf{W} \mathbf{X}_{i}\right)^{-1}\left(\mathbf{X}_{r}^{T} \mathbf{W} \mathbf{d}_{r}+\mathbf{X}_{i}^{T} \mathbf{W} \mathbf{d}_{i}\right)
$$

For many applications, the matrix $\mathbf{X}_{r}^{T} \mathbf{W} \mathbf{X}_{r}+\mathbf{X}_{i}^{T} \mathbf{W} \mathbf{X}_{i}$ in eqn. 8 is usually a Toeplitz or a block Toeplitz matrix. Therefore, only a few elements of this matrix need to be computed and eqn. 8 can be performed using an efficient algorithm as shown in [9]. Hence the required computational load can be reduced significantly.

\section{Proposed design technique}

\subsection{Initial numerator and denominator}

Here, we consider the original design problem shown in eqn. 3. The proposed design technique is based on an iterative process to find the optimal numerator and denominator for eqn. 3. At the initial step, suppose that we have chosen an initial guess with polynomial $F(z)$ and coefficients $\left\{1, f_{1}, f_{2}, \ldots, f_{N}\right\}$ through the use of the balanced model reduction algorithm presented in [10] for the denominator. The initial lattice system $B_{N}{ }^{0}(z)$ with reflection coefficients $\left\{k_{1}{ }^{0}, k_{2}{ }^{0}, \ldots, k_{N}{ }^{0}\right\}$ corresponding to $F(z)$ can be found since there exists a one-to-one correspondence between $\left\{1, f_{1}, f_{2}, \ldots, f_{N}\right\}$ and $\left\{k_{1}{ }^{0}, k_{2}{ }^{0}, \ldots, k_{N}^{0}\right\}$ [11]. From eqn. 3 , the best solution for the corresponding numerator $A^{0}(z)$ can be obtained by solving the following LCCA problem,

$$
\text { minimise } \max \left|W_{r}\left(e^{j \omega_{i}}\right)\left(D\left(e^{j \omega_{i}}\right)-\frac{A\left(e^{j \omega_{i}}\right)}{B_{N}^{0}\left(e^{j \omega_{i}}\right)}\right)\right| \forall \omega_{i} \in S_{d}
$$

Let $\mathbf{X}^{1}$ be a complex $L \times(M+1)$ matrix with its $(m$, n)th element given by

$X^{1}(m, n)=W_{r}\left(e^{j \omega_{m}}\right) \frac{\left(e^{-j \omega_{m}(n-1)}\right.}{B_{N}^{0}\left(e^{j \omega m}\right)} \quad 1 \leq m \leq L, 1 \leq n \leq M+1$

and $\mathbf{d}^{1}$ be a complex $L \times 1$ vector with its $m$ th element given by

$$
d_{m}^{1}=W_{r}\left(e^{j \omega_{m}}\right) D\left(e^{j \omega_{m}}\right) \quad 1 \leq m \leq L
$$

then the optimal coefficient vector $\mathbf{a}^{1}=\left[a_{0}{ }^{0}, a_{1}{ }^{0}, \ldots\right.$, $\left.a_{M}{ }^{0}\right]^{T}$ of $A^{0}(z)$ for eqn. 10 can be found by minimising $\left\|\mathbf{X}^{1} a^{1}-\mathbf{d}^{1}\right\|$. This minimisation problem can be solved 
by utilising the WLS algorithm. After finding the appropriate initial guess $H^{0}(z)=A^{0}(z) / B_{N}{ }^{0}(z)$, we present an iterative procedure step by step for computing $A(z)$ and $B_{N}(z)$ during the design process.

\subsection{Iterative procedure (Procedure 1)}

Step 1: At the $p$ th iteration, the Hessian matrix $\mathbf{H}=[\nabla$ $\left.H^{p}\left(e^{j \omega_{i}}\right)\right]$ is computed, where $\nabla$ represents the gradient operator $\left[\partial / \partial k_{1}, \partial / \partial k_{2}, \ldots, \partial / \partial k_{N}, \partial / \partial a_{0}, \partial / \partial a_{1}, \ldots, \partial / \partial a_{M}\right]$ and $i=1,2, \ldots, L$. We have derived the following equations for computing $\mathbf{H}$ :

$$
\begin{aligned}
& \frac{\partial H^{p}\left(e^{j \omega}\right)}{\partial a_{m}}=\frac{\partial}{\partial a_{m}}\left(\frac{A^{p}\left(e^{j \omega}\right)}{B_{N}^{p}\left(e^{j \omega}\right)}\right)=\frac{1}{B_{N}^{p}\left(e^{j \omega}\right)} \frac{\partial A^{p}\left(e^{j \omega}\right)}{\partial a_{m}}, m=0,1, \ldots, M \\
& \frac{\partial H^{p}\left(e^{j \omega}\right)}{\partial a_{m}}=\frac{\partial}{\partial k_{n}}\left(\frac{A^{p}\left(e^{j \omega}\right)}{B_{N}^{p}\left(e^{j \omega}\right)}\right)=\frac{-A^{p}\left(e^{j \omega}\right)}{\left(B_{N}^{p}\left(e^{j \omega}\right)\right)^{2}} \frac{\partial B_{N}^{p}\left(e^{j \omega}\right)}{\partial k_{n}}, n=1,2, \ldots, N
\end{aligned}
$$

Step 2: Use a linearisation scheme to approximate the frequency response error $E^{p}\left(e^{j \omega}\right)=D\left(e^{j \omega}\right)-H^{p}\left(e^{j \omega}\right)$ due to a perturbation in the coefficient vectors in the linear subspace spanned by the Hessian matrix $\mathbf{H}$. That is, the approximation error $E_{a}{ }^{P}\left(e^{j \omega}\right)=D\left(e^{j \omega}\right)-H^{P}\left(e^{j \omega}\right)-\mathbf{H v}$ is computed, where the vector $\mathbf{v}=[\Delta K, \Delta A]=\left[\Delta k_{1}, \Delta k_{2}\right.$, $\left.\ldots, \Delta k_{N}, \Delta a_{0}, \Delta a_{1}, \ldots, \Delta a_{M}\right]^{T}$ contains the increments of the independent coefficients to be found.

Step 3: Solve the following optimisation problem by utilising the WLS algorithm,

minimise $\left\|W_{r}\left(e^{j \omega_{i}}\right)\left(D\left(e^{j \omega_{i}}\right)-H^{p}\left(e^{j \omega_{i}}\right)-\mathbf{H v}\right)\right\|$

to obtain the increment coefficient vector $\mathbf{v}$.

Step 4: Perform a line search by using the NelderMeade simplex algorithm of [12] to find the best step size $t$ to update the numerator and denominator of $H^{P}(z)$ such that the following cost function is minimised:

$$
\max \left|W_{r}\left(e^{j \omega_{i}}\right)\left(D\left(e^{j \omega_{i}}\right)-\frac{A^{p}\left(e^{j \omega_{i}}\right)+t \delta A\left(e^{j \omega_{i}}\right)}{B_{N}^{p+1}\left(e^{j \omega_{i}}\right)}\right)\right| \forall \omega_{i} \in S_{d}
$$

subject to the constraint of $\max \left|k_{j}^{p}+t \Delta k_{j}\right|<k_{\max }$, where $k_{\max }$ denotes the preset maximal absolute value and must be $<1$ for the reflection coefficients in order to ensure the stability of the designed recursive digital filter. $k_{j}^{p}$ represents the $j$ th reflection coefficient obtained at the $p$ th iteration. The stability constraint of $k_{\max }<1$ in general results in that the optimal $t$ which offers a stable filter may not equal the optimal $t$ obtained by performing the line search without the stability constraint. Further, in eqn. 14, we point out that the increment polynomial $\delta A(z)$ for the numerator has the coefficient vector $\triangle A$, while the updated denominator $B_{N}^{p+1}(z)$ has the reflection coefficient vector given by $\mathbf{K}+t \Delta \mathbf{K}$.

Step 5: To alleviate the effect of the stability constraint on the optimality of the designed recursive filter, we recompute the optimal numerator for the updated denominator $B_{N}^{p+1}(z)$ by using the WLS algorithm to solve the following optimisation problem:

minimise $\left(\max \left|W_{r}\left(e^{j \omega_{i}}\right)\left(D\left(e^{j \omega_{i}}\right)-\frac{\hat{A}\left(e^{j \omega_{i}}\right)}{B_{N}^{p+1}\left(e^{j \omega}\right)}\right)\right|\right) \forall \omega_{i} \in S_{d}$

Step 6: Set the updated numerator $A^{p+1}(z)$ equal to $\hat{A}(z)$ obtained from eqn. 15 and compute the corresponding frequency response error $E^{p+1}\left(e^{j \omega}\right)=D\left(e^{j \omega}\right)_{-}$ $H^{p+1}\left(e^{j \omega}\right)$.
Step 7: Compute the ratio $\left(\left\|W_{r}\left(e^{j \omega_{i}}\right) E^{p+1}\left(e^{j \omega_{i}}\right)\right\|-\right.$ $\left.\left\|W_{r}\left(e^{j \omega_{i}}\right) E^{p}\left(e^{j \omega_{i}}\right)\right\|\right) /\left\|W_{r}\left(e^{j \omega_{i}}\right) E^{p}\left(e^{j \omega_{i}}\right)\right\|$. If this ratio is less than a preset positive number $\kappa$, then the design procedure is terminated. Otherwise, we continue this procedure and go to step 1.

Remarks: There are two situations where the Hessian matrix $\mathbf{H}$ may degenerate.

Case 1: The columns of $\mathbf{H}$ are not linearly independent. Then the optimal solution for eqn. 13 will not be unique. To find an appropriate optimal solution, we construct a matrix $\mathbf{G}$ by choosing the independent columns from $\mathbf{H}$ and a vector $\mathbf{u}$ by choosing the components of $\mathbf{v}$ corresponding to the independent columns. Then use $\mathbf{G}$ and $\mathbf{u}$ to replace $\mathbf{H}$ and $\mathbf{v}$ of eqn. 13 .

Case 2: At the $p$ th iteration, the $i$ th reflection coefficient $k_{j}$ may have the absolute value equal to $k_{\max }$. To tackle this difficulty, we construct a vector $\mathbf{u}$ by eliminating $\Delta k_{j}$ of the vector $\mathbf{v}$ and a matrix $\mathbf{G}$ by eliminating the column of $\mathbf{H}$ corresponding to $\Delta k_{j}$. Then we use the $\mathbf{G}$ and $\mathbf{u}$ to replace $\mathbf{H}$ and $\mathbf{v}$ of eqn. 13 .

\section{Design examples}

In this Section, several computer simulations of designing recursive filters with arbitrary magnitude and phase responses are presented for illustrating the effectiveness of the proposed technique. All the simulations were performed on a 80486 PC using a MATLAB programming language. The value of $\kappa$ used for terminating procedure 1 was set to $10^{-3}$. For each of the design examples, the WLS algorithm was used for solving the Chebysshev solution of the resulting LCCA approximation problem and the value of $k_{\max }$ was set to 0.98 . The number $L$ of frequency grid points used during the design process was set to 300 . Moreover, the weighting function $\left.W_{r}\left(e^{j \omega_{i}}\right)\right)$ was set to 1 in both the passband and stopband for examples 1 and 3 , and was set to 1 and 3 in the passband and stopband, respectively, for example 2.

Example 1. A lowpass recursive filter of order 4/4: The specifications for this design are exactly the same as those of example 1 presented in [4]. The passband and stopband are set to the ranges $[0,0.1]$ and $[0.2,0.5]$, respectively. The desired group delay is set to 5 . Some significant design results obtained by utilising the proposed technique are listed in Table 1. For comparison, we also present the design results of using the technique of [4]. Table 2 shows the filter coefficients, while Figs. 2-4 plot the magnitude, group delay and approximation error responses of the filter designed by using the proposed technique. We note from Table 1 that the proposed technique outperforms the technique of [4] in this case.

Table 1: Performance comparison for example 1

\begin{tabular}{lll}
\hline & $\begin{array}{l}\text { Proposed } \\
\text { algorithm }\end{array}$ & $\begin{array}{l}\text { Algorithm } \\
\text { of [4] }\end{array}$ \\
\hline IIR filter order & $4 / 4$ & $4 / 4$ \\
Chebȳshev error in passband & 0.0215 & 0.0420 \\
Chebȳshev error in stopband & 0.0215 & 0.0420 \\
$\begin{array}{l}\text { Chebȳshev error in stopband, dB } \\
\text { Range of passband group delay }\end{array}$ & -33.35 & -27.50 \\
$\begin{array}{l}\text { Max. variation of passband group } \\
\text { delay }\end{array}$ & 0.57 & $4.65-6.34$ \\
\hline
\end{tabular}


Table 2: Designed filter coefficients for example 1

\begin{tabular}{lll}
\hline$i$ & $a_{i}$ & \multicolumn{1}{l}{$k_{i}$} \\
\hline 0 & $-2.4874193 \times 10^{-2}$ & \\
1 & $4.6554285 \times 10^{-2}$ & $-8.4567002 \times 10^{-1}$ \\
2 & $3.8891673 \times 10^{-3}$ & $8.7923704 \times 10^{-1}$ \\
3 & $1.7479002 \times 10^{-2}$ & $-7.8745132 \times 10^{-1}$ \\
4 & $4.1137105 \times 10^{-2}$ & $3.9254935 \times 10^{-1}$ \\
\hline
\end{tabular}

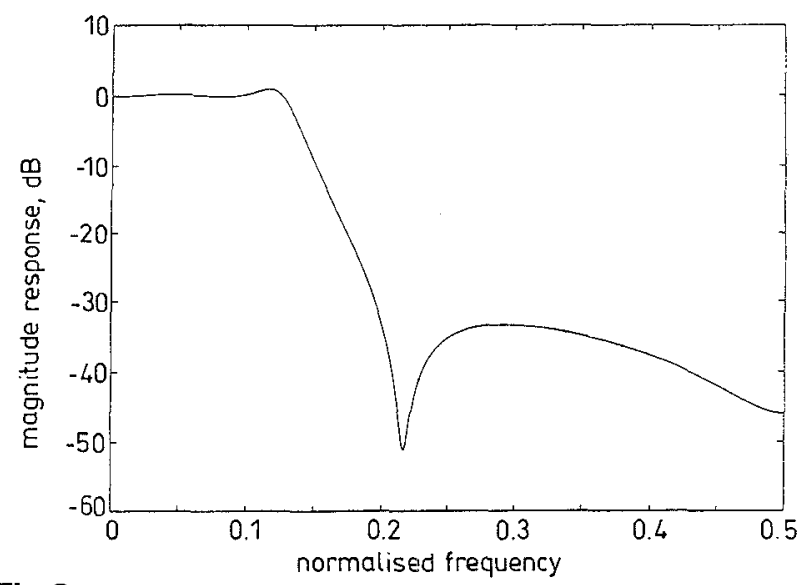

Fig.2 Magnitude response of designed filter for example 1

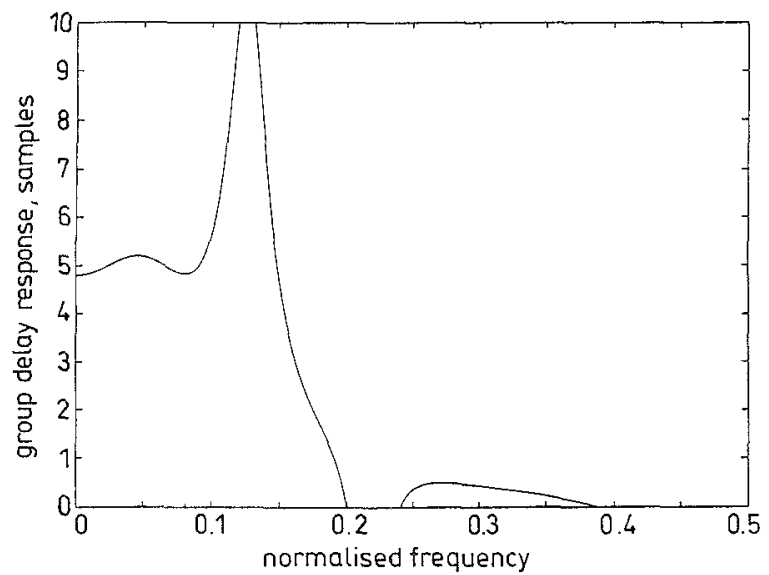

Fig.3 Group delay response for example 1

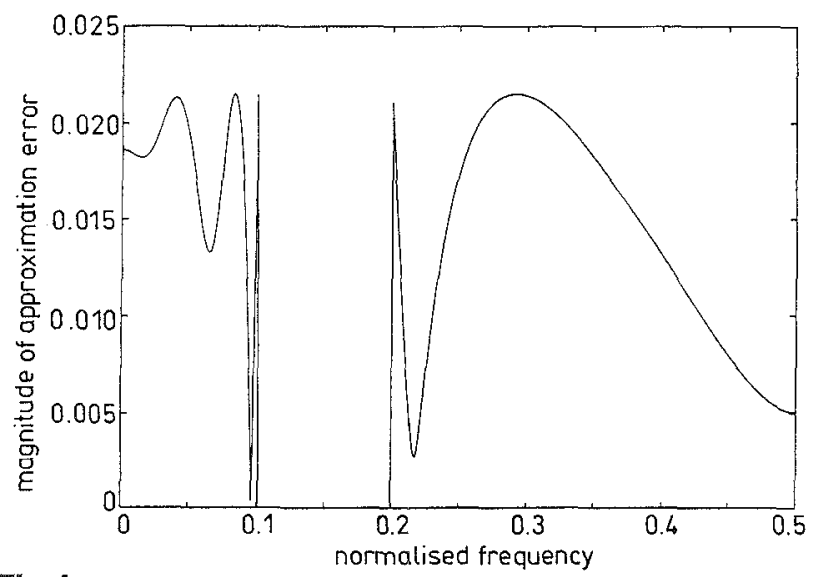

Fig.4 Approximation error for example 1

Example 2. A lowpass recursive filter of order 6/7: For this design, the specifications are exactly the same as those of example 2 presented in [4]. The passband and stopband are set to the ranges $[0,0.1]$ and $[0.2,0.5]$, respectively. The desired group delay is set to 10 . Some significant design results obtained by utilising the proposed technique are listed in Table 3. Table 4 shows the filter coefficients, while Fig. 3 plots the magnitude, group delay and approximation error responses of the designed filter. For comparison, the significant filter performances of using the technique presented in [4] are also shown in Table 3. Again, we note from Table 3 that the proposed technique outperforms the technique of [4].

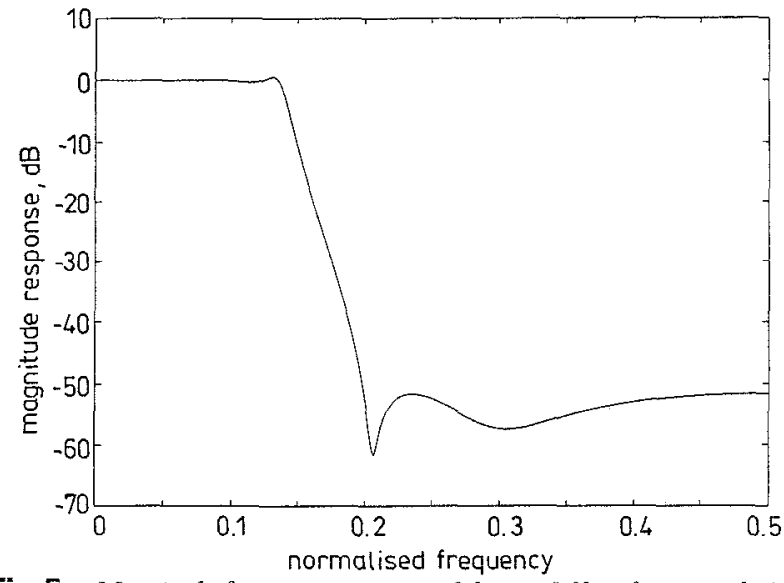

Fig.5 Magnitude frequency response of designed filter for example 2

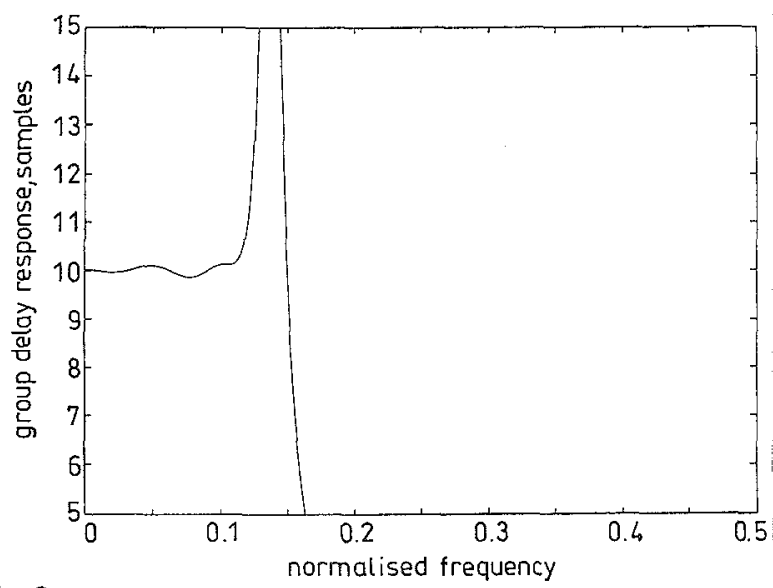

Fig.6 Group delay response for example 2

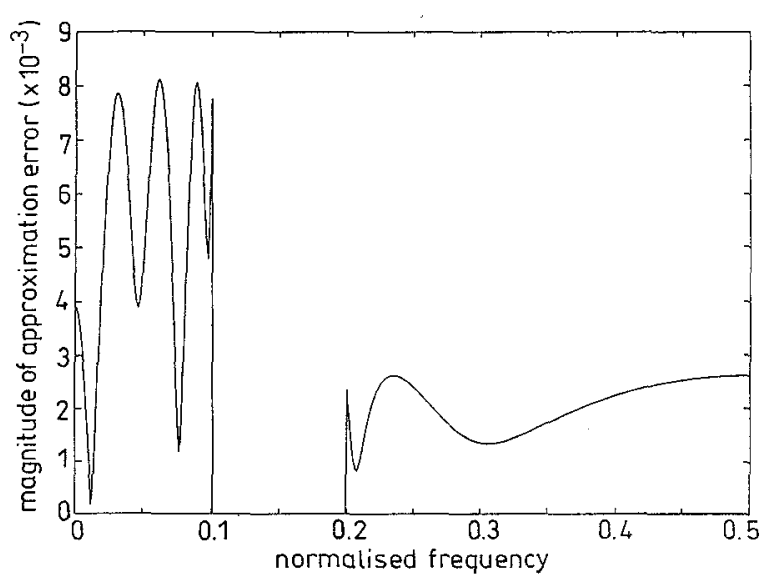

Fig.7 Approximation error for example 2

Example 3. A wideband linear-phase lowpass recursive filter of order 18/18: For this case, the chosen design specifications are exactly the same as those of example 6.1 presented in [13]. The passband and stopband are set to the ranges $[0,0.25]$ and $[0.3,0.5]$, respectively. 
Table 3: Performance comparison for example 2

\begin{tabular}{lll}
\hline & $\begin{array}{l}\text { Proposed } \\
\text { algorithm }\end{array}$ & $\begin{array}{l}\text { Algorithm } \\
\text { of [4] }\end{array}$ \\
\hline IIR filter order & $6 / 7$ & $6 / 7$ \\
Chebȳshev error in passband & 0.0081 & 0.0155 \\
Chebȳshev error in stopband & 0.0026 & 0.0052 \\
$\begin{array}{l}\text { Chebȳshev error in stopband, dB } \\
\text { Range of passband group delay }\end{array}$ & -51.6 & -45.7 \\
$\begin{array}{l}\text { Max. variation of passband group } \\
\text { delay }\end{array}$ & 0.14 & 0.86 .10 .11 \\
\hline
\end{tabular}

Table 4: Designed filter coefficients for example 2

\begin{tabular}{lll}
\hline$i$ & \multicolumn{1}{l}{$a_{i}$} & \multicolumn{1}{c}{$k_{i}$} \\
\hline 0 & $3.8657742 \times 10^{-3}$ & \\
1 & $-1.1035528 \times 10^{-2}$ & $-8.7846678 \times 10^{-1}$ \\
2 & $1.5855231 \times 10^{-2}$ & $8.8107956 \times 10^{-1}$ \\
3 & $-2.4540573 \times 10^{-2}$ & $-8.9103741 \times 10^{-1}$ \\
4 & $3.0113662 \times 10^{-2}$ & $8.8192088 \times 10^{-1}$ \\
5 & $-2.0369372 \times 10^{-2}$ & $-8.3376837 \times 10^{-1}$ \\
6 & $1.5996173 \times 10^{-2}$ & $6.4279357 \times 10^{-1}$ \\
7 & & $-2.3076139 \times 10^{-1}$ \\
\hline
\end{tabular}

Table 5: Performance comparison for example 3

\begin{tabular}{lll}
\hline & $\begin{array}{l}\text { Proposed } \\
\text { algorithm }\end{array}$ & $\begin{array}{l}\text { Algorithm } \\
\text { of [13] }\end{array}$ \\
\hline IIR filter order & $18 / 18$ & $18 / 18$ \\
Chebȳshev error in passband & 0.0027 & 0.0405 \\
Chebȳshev error in stopband & 0.0027 & 0.0097 \\
$\begin{array}{l}\text { Chebȳshev error in stopband, dB } \\
\text { Range of passband group delay }\end{array}$ & -51.41 & -40.24 \\
$\begin{array}{l}\text { Max. variation of passband group } \\
\text { delay }\end{array}$ & 0.07 & 0.26 \\
\hline
\end{tabular}

\section{Table 6: Designed filter coefficients for example 3}

\begin{tabular}{|c|c|c|}
\hline$i$ & $a_{i}$ & $k_{i}$ \\
\hline 0 & $-1.4179658 \times 10^{-4}$ & \\
\hline 1 & $-8.9369242 \times 10^{-4}$ & $-7.7943336 \times 10^{-1}$ \\
\hline 2 & $7.6111604 \times 10^{-3}$ & $6.3921350 \times 10^{-1}$ \\
\hline 3 & $-2.6017086 \times 10^{-2}$ & $-5.8972093 \times 10^{-1}$ \\
\hline 4 & $5.7879648 \times 10^{-2}$ & $6.0054235 \times 10^{-1}$ \\
\hline 5 & $-9.6967825 \times 10^{-2}$ & $-6.2268220 \times 10^{-1}$ \\
\hline 6 & $1.3348250 \times 10^{-1}$ & $6.3505035 \times 10^{-1}$ \\
\hline 7 & $-1.6271720 \times 10^{-1}$ & $-6.3761634 \times 10^{-1}$ \\
\hline 8 & $1.8723776 \times 10^{-1}$ & $6.3439276 \times 10^{-1}$ \\
\hline 9 & $-2.1098236 \times 10^{-1}$ & $-6.2774302 \times 10^{-1}$ \\
\hline 10 & $2.3590591 \times 10^{-1}$ & $6.1804955 \times 10^{-1}$ \\
\hline 11 & $-2.6525684 \times 10^{-1}$ & $-6.0394533 \times 10^{-1}$ \\
\hline 12 & $3.0120905 \times 10^{-1}$ & $5.8174536 \times 10^{-1}$ \\
\hline 13 & $-3.3170910 \times 10^{-1}$ & $-5.4369487 \times 10^{-1}$ \\
\hline 14 & $3.2903791 \times 10^{-1}$ & $4.7616400 \times 10^{-1}$ \\
\hline 15 & $-2.7820673 \times 10^{-1}$ & $-3.6507023 \times 10^{-1}$ \\
\hline 16 & $2.0197693 \times 10^{-1}$ & $2.2013488 \times 10^{-1}$ \\
\hline 17 & $-1.3308297 \times 10^{-1}$ & $-9.0921627 \times 10^{-2}$ \\
\hline 18 & $6.7461232 \times 10^{-2}$ & $2.0165519 \times 10^{-2}$ \\
\hline
\end{tabular}

The passband possesses a constant group delay equal to 22 . The significant design results obtained by utilising the proposed technique are presented in Table 5.
Table 6 shows the filter coefficients, while Fig. 4 plots the magnitude, group delay and approximation error responses of the designed filter. For comparison, the design results of using the technique of [13] are also shown in Table 5 . We note that the filter designed by the proposed technique possesses better performance than that designed by the technique of [13].

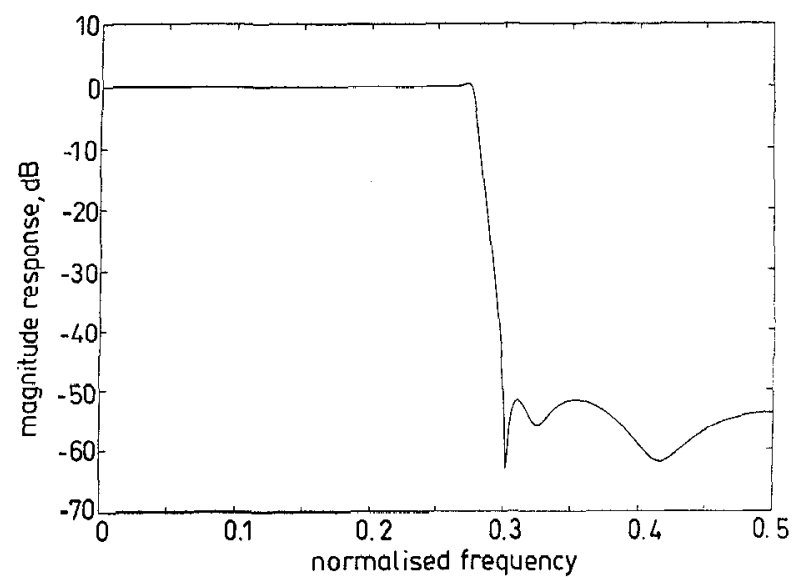

Fig. 8 Magnitude frequency response of designed filter for example 3

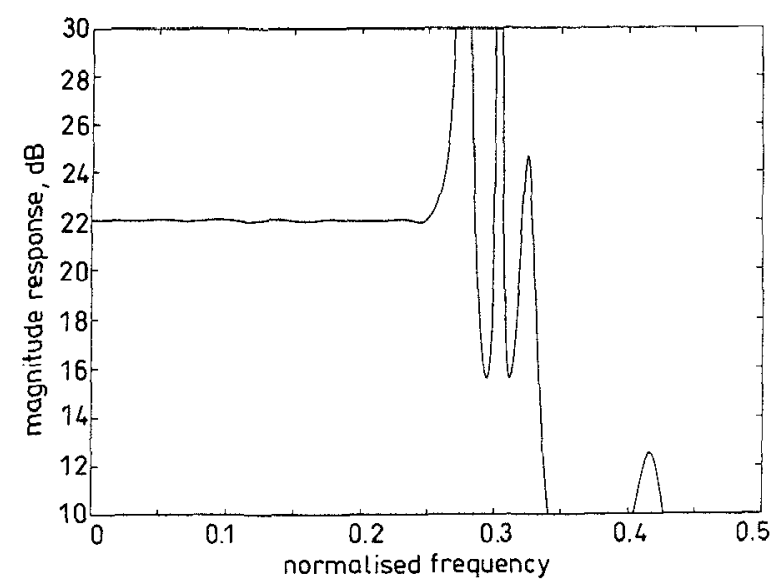

Fig.9 Group delay response for example 3

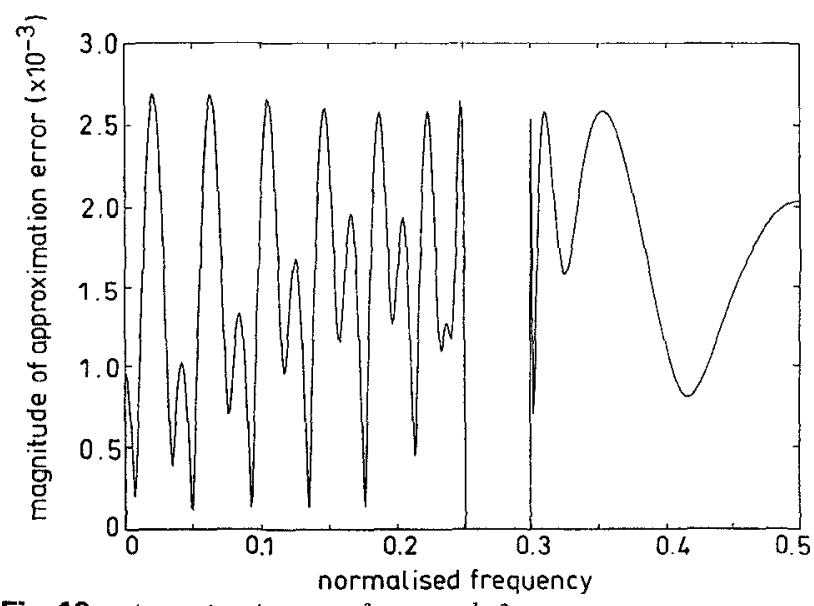

Fig. 10 Approximation error for example 3

\section{Conclusion}

This paper has presented an efficient technique based on a WLS algorithm for the minimax design of recursive digital filters with a lattice denominator. At each iteration of the proposed technique, the core work includes appropriately adjusting the tap coefficients of the numerator and the reflection coefficients for the 
denominator to reduce the resulting Chebyshev error and keep the designed filter stable. For the first task, the WLS algorithm presented in [7] is utilised to solve the required linear complex Cheby shev approximation (LCCA) problems. For the second task, the stability of the designed recursive filter is ensured by incorporating an efficient stabilisation procedure to make all of the reflection coefficient values fall between -1 and +1 . Computer simulations have shown that the proposed technique provides more satisfactory design results than existing design techniques.

\section{Acknowledgment}

This work was supported by the National Science Council under grant NSC85-2213-E002-027.

\section{References}

1 CHOTTERA, A.T., and JULILIEN, G.A.: 'A linear programming approach to recursive filters with linear phase', IEEE Trans. Circuits Syst., 1982, CAS-29, pp. 139-149

2 CORTELAZZO, G., and LIGHTNER, M.R.: 'Simultaneous design in both magnitude and delay of IIR and FIR filters based on multiple criterion optimization', IEEE Trans. Acoust. Speech Signal Process., 1984, ASSP-32, pp. 949-967

3 POWELL, M.J.D.: 'A fast algorithm for nonlinearly constrianed optimization calculations', in DOLD, A and ECKMAN, B. Eds.): Proc. Dundee Conf. Num. anal. (Lecture Notes in Mathematics), (Springer-Verlag, New York, 1977)
4 CHEN, X., and PARKS, T.W.: 'Design of IIR filters in the complex domain', IEEE Trans. Acoust. Speech Signal Process., 1990, ASSP-38, pp. 910-920

5 CHEN, $X$, and PARKS, T.W.: 'Design of FIR filters in the complex domain', IEEE Trans. Acoust. Speech Signal Process., 1987, ASSP-35, pp. 144-153

6 ELLACOTT, S., and WILLIAMS, J.: 'Rational Chebyshev approximation in the complex plane', SIAM J. Numer. Anal., $1976,13,(3)$, pp. 310-323

7 LIM, Y.C., LEE, J.-H., CHEN, C.-K., and YANG, R.H.: 'A weighted least squares algorithm for quasi-equiripple FIR and IIR digital filter design', IEEE Trans. Signal Process., 1992, 40, pp. $551-558$

8 LIM, Y.C.: 'On the synthesis of IIR digital filters derived from AR lattice network', IEEE Trans. Acoust. Speech Signal Process., 1984, ASSP-32, pp. 741-749

9 LEE, J.-H., CHEN, C.-K., and LIM, Y.C.: 'Design of discrete coefficient FIR digital filters with arbitrary amplitude and phase responses', IEEE Trans. Circuits Syst., Part II: Analog Digit. Signal Process., 1993, 40, pp. 444 448

10 BELICZYNSKI, B., KALE, I., and CAIN, G.D.: 'Approximation of FIR by IIR digital filters: an algorithm based on balanced model reduction', IEEE Trans. Signal Process., 1992, 40, pp. 532-542

11 OPPENHEIM, A.V., and SCHAFFER, R.W.: 'Discrete-time signal processing' (Prentice-Hall, Englewood Cliffs, New Jersey, 1989)

12 NELDER, J.A., and MEADE, R.: 'A simplex method for function minimization', Comput. J., 1965, 7, pp. 308-313

$13 \mathrm{GU}, \mathrm{G}$., and SHENOI, B.A.: 'A novel approach to the synthesis of recursive digital filters with linear phase', IEEE Trans. Circuits Syst., 1991, CAS-38, pp. 602-612 